\title{
A Study of various soft computing techniques for iris recognition
}

\author{
Gaganpreet kaur $^{1}$, Dilpreet kaur ${ }^{2}$, Dr. Dheerendra Singh ${ }^{3}$ \\ ${ }^{1}$ A.P., Deptt. Of C.S.E., SGGSWU, Fatehgarh Sahib, Punjab, India. \\ ${ }^{2}$ Research Scholar, Deptt. Of C.S.E., SGGSWU, Fatehgarh Sahib, Punjab, India. \\ ${ }^{3}$ Prof \& Head, SUSCET, Tangori, Punjab, India.
}

\begin{abstract}
Iris Recognition is considered as one of the best biometric method used for human identification and verification, because of its unique feature that differ from one person to another, and its importance in the security field. This paper introduced the two soft computing techniques of neural network which are used for pattern matching in iris recognition. This paper represents iris recognition based Competitive neural network Learning Vector Quantization (LVQ) and Adaptive Resonance Associative Map (ARAM) with essential redefinition of pattern similarity and also introduces the mixture model ARAM for the measurements of patterns. Keywords - ARAM, Biometric, Iris Recognition, LVQ, and Mixture model ARAM, Neural Network.
\end{abstract}

\section{INTRODUCTION}

Iris recognition is a method of biometric authentication that uses pattern-recognition techniques based on high resolution images of the irises of an individual's eyes. Not to be confused with another, less prevalent, ocularbased technology, retina scanning, and iris recognition uses camera technology, with subtle infrared illumination reducing specular reflection from the convex cornea, to create images of the detail-rich, intricate structures of the iris. Converted into digital templates, these images provide mathematical representations of the iris that yield unambiguous positive identification of an individual. Iris recognition efficacy is rarely impeded by glasses or contact lenses. Iris technology has the smallest outlier group of all biometric technologies. The only biometric authentication technology designed for use in a one-to-many search environment, a key advantage of iris recognition is its stability, or template longevity, as, barring trauma, a single enrollment can last a lifetime. Plemmons proposed different optimization methods for designing the optics and the image processing algorithms, and provide laboratory and simulation results from applying these systems and results on restoring the intermediate phase encoded images using both direct Wiener filter and iterative conjugate gradient method [1].

Christel-Loïc Tisse et al examined a new iris recognition system that implements (i) gradient decomposed Hough transform / integral-differential operators combination for iris localization and (ii) the "analytic image" concept to extract pertinent information from iris texture [2]. Li Ma et al proposed a new approach for personal identification based on iris recognition. The body of their papers details the steps of iris recognition, including image preprocessing, feature extraction and classifier design. The proposed algorithm uses a bank of Gabor filters to capture both local and global iris characteristics to form a fixed length feature vector. Iris matching is based on the weighted Euclidean distance between the two corresponding iris vectors and is therefore very fast [3]. Narayanswamy et al employs an especially designed Wave front Coded lens customized for iris recognition. They presented experimental results that show the benefits of this technology for biometric identification [4]. Zhuoshi Wei et al presented a framework to synthesize large realistic iris databases, providing an alternative to iris database collection. Firstly, iris patch is used as a basic element to characterize visual primitive of iris texture, and patch-based sampling is applied to create an iris prototype [5]. Czajka et al presents an original, independent of typical cryptographic techniques, method of biometric replay attack prevention for iris biometrics. The proposed solution takes advantage of appropriate enhancements of iris templates and relies on appropriate data alteration within the image space resulting in significant changes of the resulting code in the iris feature space [6].

\section{IRIS PREPROCESSING}

Iris preprocessing consider following stages:

\subsection{Localization}




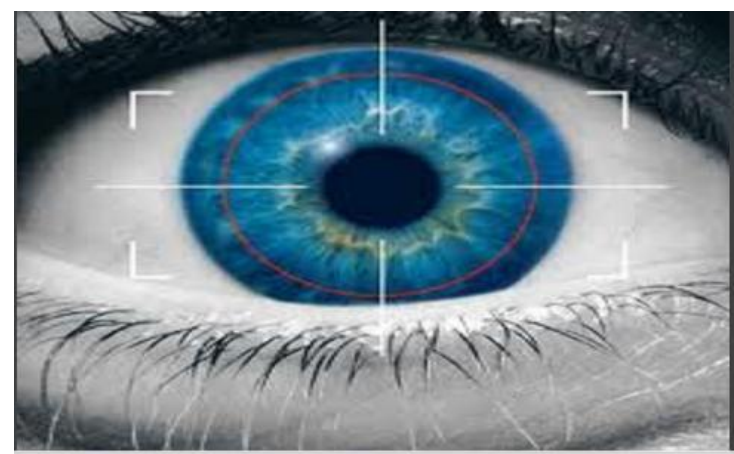

Fig1. Localization of iris

The first step in the localization process is pupil segmentation based on analysis of the histogram. It is assumed, like in [7] that a pupil is the darkest area in the image and standard deviation of luminance in this area is relatively low. It has to be emphasized that unwanted elements may appear in the threshold image. Nevertheless pupil can be segmented by relatively simple method, based on image thresholding using the histogram analysis.

\subsection{Segmentation}

Segmentation is one of the important steps in preprocessing since it separates the input iris image into several components making it feasible to extract features easily. The Segmentation process is defined as separating the input iris image into several components, so it is very important step in preprocessing because it describes and recognize the input image. One of the best ways to separate the pupil from the whole eye is using circular Hough transformation in order to find the circle of the pupil [7]. The pupil is the largest black area in the intensity image; its edge can be easily detected from the binary image by using a suitable threshold of the intensity image.

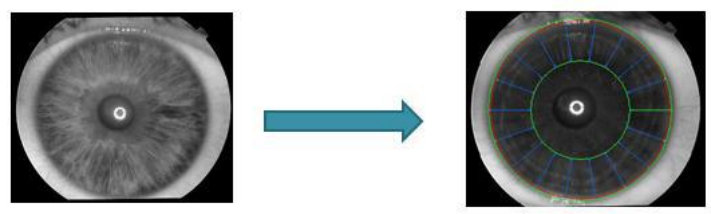

Fig2. Example of Edge Detection [7]

\subsection{Normalization}

The purpose of iris normalization is to get the same region of iris to do matching regardless pupil dilation and the different iris size caused by the different distance between the eye and video zoom factor. Moreover, the shift, accounting for the offsets of the eye in the plane parallel to the camera's sensor, should also be eliminated [8]. Wildes [9][10] used an image-registration technique to eliminate dilation, scaling and rotation. This approach wraps a new iris image into alignment with a normal image according to a mapping function so that the difference between the two iris images is minimal.

\subsection{Feature Vector Extraction and Match}

Feature selection and extraction is to find out the important features to do matching. The visible features of an iris are ciliary processes, contraction furrows, crypts, rings, corona, and freckle [11] and so on. How to set a model to extract the feature of different irises and match them is especially important for it determines the results of the whole system directly [12] [13].

\section{NEURAL NETWORK}

The term "neural network" [15] was traditionally used to refer to a network or circuit of biological neurons. The modern usage of the term often refers to artificial neural networks, which are composed of artificial neurons or nodes. Thus the term has two distinct usages:

i) "Biological neural networks" are made up of real biological neurons that are connected or functionally related in the peripheral nervous system or the central nervous system. In the field of neuroscience, they are often identified as groups of neurons that perform a specific physiological function in laboratory analysis.

ii) "Artificial neural networks" are composed of interconnecting artificial neurons (programming constructs that mimic the properties of biological neurons). Artificial neural networks may either be used to gain an 
understanding of biological neural networks, or for solving artificial intelligence problems without necessarily creating a model of a real biological system. The real, biological nervous system is highly complex: artificial neural network algorithms attempt to abstract this complexity and focus on what may hypothetically matter most from an information processing point of view. Good performance or performance mimicking animal or human error patterns, can then be used as one source of evidence towards supporting the hypothesis that the abstraction really captured something important from the point of view of information processing in the brain. Another incentive for these abstractions is to reduce the amount of computation required to simulate artificial neural networks, so as to allow one to experiment with larger networks and train them on larger data sets.

An artificial neural network usually called neural network [15], is a mathematical model or computational model that is inspired by the structure and functional aspects of biological neural networks. A neural network consists of an interconnected group of artificial neurons, and it processes information using a connectionist approach to computation. In most cases an ANN is an adaptive system that changes its structure based on external or internal information that flows through the network during the learning phase. Modern neural networks are non-linear statistical data modeling tools. They are usually used to model complex relationships between inputs and outputs or to find patterns in data.

\subsection{Soft computing techniques of neural network}

Following two approaches of the neural network are used for enhancing the security in iris recognition system:

\subsubsection{Learning Vector Quantization (LVQ)}

Learning Vector Quantization is a prototype-based supervised classification algorithm. LVQ can be understood as a special case of an artificial neural network. LVQ is one of the competitive neural networks. LVQ is a pattern classification method; each output node is represented as a class. The weight vector of an output node is called a reference or codebook vector $(\mathrm{CV})$. The LVQ attempts to adjust the weights to approximate a theoretical bayes classifier. The result obtained depends on the majority voting among several weak classifiers Input vectors are classified by assigning them as a class label of the weight vector closest to the input vector[14] [15]. The result obtained depends on the majority voting among several weak classifiers. The LVQ consisting of two layers; the first layer is the input layer which contains the input neurons, while the second layer is the output layer that contains output neurons.

The final step is the pattern matching of the Iris image with the stored templates from the database. The Following figure shows Pattern matching process. These patterns are used to create templates forlris recognition. It will work fine for less number of users, but it is not recommended for large number of users since it requires large storage memory.

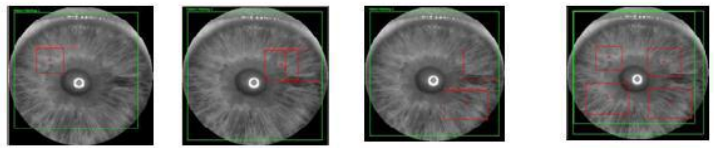

Fig3. Pattern matching process

\subsubsection{Template matching result}

The Iris recognition steps, feature extraction, Pattern matching are performed using NI Vision Assistant and NI Lab VIEW. The NI vision script for iris recognition using template matching.

\begin{tabular}{|l||l|}
\hline Results .... & 1 \\
\hline \hline X Position & 144.000000 \\
\hline Y Position & 366.50000 \\
\hline Angle & 0.000000 \\
\hline Score & 1000.00000 \\
\hline
\end{tabular}

Fig4. Result of template matching

\subsubsection{Adaptive Resonance Associative Map (ARAM)}

The Adaptive Resonance Associative Map (ARAM) [16] belongs to the family of Adaptive Resonance Theory (ART) self-organizing neural networks. Like another member of the family, ART-MAP ARAM is capable of incrementally learning recognition categories and multidimensional maps of patterns. Compared to ART-MAP, ARAM contains as implified pattern matching and learning process. An ARAM network has two individual 
short term memory (STM) and a shared long term memory (LTM) 1 that encodes the associated knowledge from the two feature fields. The learning of the network is guided by an orienting subsystem with two logical gates, defined with two vigilance parameter. The logical gates conditionally switch and reset the network state according to predefined rules, and hence affect knowledge encoding in the LTM. ARAM acquires its domain knowledge through an online, hard competitive learning process.

ARAM uses two functions (choice and match) to evaluate the similarity between the input and recognition category. These two functions may or may not have same definition, optionally providing a different view to conform the degree of pattern matching. Secondly, the use of vigilance thresholds ensures only significantly similar patterns may be grouped together. On the other hand, the vigilance parameters primarily affect the clustering process. Lower vigilance thresholds generally lead to fewer recognition categories, and hence rougher clustering result. Lastly while most importantly, ARAM provides an effective infrastructure for learning of associative knowledge from two different domains. Depending on the input signals, ARAM may be applied to different learning tasks. Examples include text and document classification [17], clustering and personalized knowledge management and association rule mining [18] [19].

\subsubsection{Mixture Model ARAM for GO Annotation and Gene Expression Data}

whereas Mixture Model ARAM is straightforward: for each gene product. In ARAM's pattern field A to encode its expression profile and B to encode its GO annotation. Following common practices, the expression profile is presented in vector format, and the GO annotation is presented as a set of descriptive GO terms, denoted as: $\mathrm{x}=$ $(\mathrm{xa} \mid \mathrm{xb})=\left(\mathrm{e}^{--} \mathrm{x} \rightarrow \mathrm{p} \mid\right.$ go terms $\left.\}\right)$. The LTM recognition category encodes an associative pattern $\mathrm{w}=(\mathrm{wa} \mid \mathrm{wb})$, where wa and wb respectively are the expression pattern and GO annotation term(s) representative to the inputs that form the corresponding category [20].

\subsubsection{Pattern Field for Gene Expression}

Appropriate normalization of gene expression data prevents category proliferation without compromising their biological representation. Variations of normalization techniques are adopted in different conditions [21][22]. Thus the Mixture Model ARAM network does not contain a fixed normalization link to alter the original input gene expression. Input expressions are assumed and they are properly pre-normalized, and define the link between $\mathrm{Fa} 0$ and $\mathrm{Fb} 1$ as a simple feed forward copy operation: $\mathrm{xa}=\mathrm{Ia}$. The measurement of the similarity between two GO terms as the information content of their minimal subsumer. A so-called minimal subsumer of two terms ti and $\mathrm{t} \mathrm{j}$, denoted as $\nabla$ (ti, $\mathrm{t} \mathrm{j})$, is the subsumer that has the minimal likelihood. To formalize:

$$
\begin{array}{r}
\operatorname{Sim}(t \mathrm{ti}, \mathrm{t} \mathrm{j}) \equiv \mathfrak{J}(\nabla(\mathrm{ti}, \mathrm{t} \mathrm{j})) \\
=-\log (\min \{\mathrm{p}(\mathrm{t}) \mid \mathrm{t} \in \mathrm{S}(\mathrm{ti}, \mathrm{t} \mathrm{j})\})
\end{array}
$$

Where $S(t i, t j)$ is the subsumer set of term ti and $t \mathrm{j}$, essentially being their common ancestor terms.

Similarity between GO Annotations of two Genes while equation 1 measures the semantic similarity between two GO terms, it is common that a gene product may be annotated with multiple GO terms, which will lead to multiple term-to-term similarities between two genes. To formalize, suppose the multiple GO annotations of two genes products gi and gj are denoted as: $\mathrm{Ai}=\{\mathrm{ti} 1, \mathrm{ti} 2 \ldots \mathrm{tiP}\}$ and $\mathrm{Aj}=\{\mathrm{t} \mathrm{j} 1, \mathrm{t} \mathrm{j} 2 \ldots \mathrm{t} \mathrm{jQ}\}$ respectively, their similarity is then calculated as:

$$
\operatorname{Sim}(A i, A j)=\max \{\operatorname{sim}(t i x, t j y) \mid x \in[1, P], y \in[1, Q]\}
$$

By applying the maximal term-to-term similarity as the similarity between to GO annotations, we essentially identify their subsume that has the maximal information content, i.e. the maximal common factor.

\section{CONCLUSION}

This paper concluded that there are two important soft computing approaches of neural network which are used for pattern matching in iris recognition to enhance the performance. Learning Vector Quantization is a prototype-based supervised classification algorithm in which each output node is represented as a class. Codebook vector $(\mathrm{CV})$ is used for output node. It uses bayes classifier for adjusting the weight. The result obtained depends on the majority voting among several weak classifiers. The patterns matching of the Iris image is to be done with the stored templates from the database. Templates are created with these patterns but it will work fine for less number of users; it is not recommended for large number of users since it requires large storage memory whereas the Adaptive Resonance Associative Map (ARAM) belongs to the family of Adaptive Resonance Theory (ART) self-organizing neural networks. It is capable of incrementally learning recognition 
categories and multidimensional maps of patterns. It contains a simplified pattern matching and learning. To improve the pattern matching process mixture model ARAM is used which used gene ontology (GO) and gene expression for checking the similarities and measurements of various pattern. This approach reduced the noise from the whole data and provides the expression between various patterns. As compared Learning Vector Quantization, the Adaptive Resonance Associative map is more recommended for large numbers of users.

\section{REFERENCES}

[1] R. Plemmonsa, M. Horvatha, E. Leonhardta, P. Paucaa, S. Prasad, "Computational Imaging Systems for Iris Recognition", Processing of Spiel 2004.

[2] C. L. Tisse, L. Martin, L. Torres, M. Robert, "Person identification technique using human iris recognition", ST Journal of System Research Current Issue 2003.

[3] L. Ma, U. Wang, T. Tan, "Iris Recognition Based on Multichannel Gabor Filtering", The 5th Asian Conference on Computer Vision, pp.23-25, Melbourne, Australia, January 2002.

[4] R. Narayanswamy, P. E. X. Silveir, "Extended Depth-of-Field Iris Recognition System for a Workstation Environment", Proceedings of the Spied Biometric Technology for Human Identification II,Vol. 5779, pp. 41-50, Orlando, FL, 2005.

[5] Z. Wei, T. Tan and Z. Sun, "Synthesis of Large Realistic Iris Databases Using Patch-based Sampling", IEEE, 2008.

[6] A. Czajka, A. Pacut, "Replay Attack Prevention for Iris Biometrics", IEEE, ICCST 2008.

[7] C. Teo and H. Ewe, "An Efficient One-Dimensional Fractal Analysis for Iris Recognition", Proceedings of the 13thWSCGInternational Conference in Central Europe on Computer Graphics, Visualization and Computer Vision, pp. 157-160, Plzen-Bory, Czech Republic, January 31 - February 4, 2005.

[8] Daugman J G., "High confidence visual recognition of persons by a test of statistical independence", IEEE Trans. Pattern Anal. Machine Intel. 1993, vol.15, no.11, pp.1148-1161.

[9] Wildes R P., "Iris Recognition: An Emerging Biometric Technology", In Proceeding of the IEEE, Piscataway, NJ, vol. 85, pp.1348$1363,1997$.

[10] Daugman J G., "Recognition persons by their pattern", Information Security Technical report, vol.13, no.1, pp. 33-39, 1998.

[11] Daugman J G., "High confidence visual recognition of persons by a test of statistical independence", IEEE Trans. Pattern Anal. Machine Intel, 1993, vol.15, no.11, pp. 1148-1161.

[12] Daugman J G. "High Confidence Recognition of Person by Rapid Video Analysis of Iris Texture", European Convection on Security Detection and Brighton, Brighton, UK, pp. 244-251, 1995.

[13] Daugman J G. "Recognition persons by their pattern", Information Security Technical report, vol.13, no.1, pp.33-39, 1998.

[14] CBSR, 2005.Center for biometrics and security research. http://www.cbsr.ia.ac.cn/english/Databases.asp

[15] Zhou Zhiping, Hui Maomao, Sun Ziwen, “An iris recognition based on 2DWPCA and neural network", pp.9781-4244-2723, 9/09/\$25.00@ 2009, IEEE.

[16] Tan A.H., "Adaptive Resonance Associative Map for Neural Networks", Vol.8, No.3, pp. 437-446, 1995.

[17] Tan A.H., "Predictive self-organizing networks for text categorization", Proceedings of the Pacific-Asia Conference on Knowledge Discovery and Data Mining (PAKDD), pp. 66-77, 2001

[18] Tan A.H., Ong H.L., Pan H., Ng J., Li Q.X., “Towards personalized web intelligence”, Knowledge and Information Systems, Vol.6, No.5, pp.595-616, 2004.

[19] Tan A.H., Pan H., "Predictive neural networks for gene expression data analysis", Neural Networks, Vol.18, No.3, pp. 297-306, 2005.

[20] Carpenter G., Gross berg S., Rosen D., "ART 2-A: An adaptive resonance algorithm for rapid category learning and recognition", Neural Networks, Vol.4, pp. 493-504, 1991.

[21] The Gene Ontology Consortium, "Gene ontology: tool for the unification of biology", Nature Genetics, Vol.25, No.1, pp. 25-29, 2000.

[22] Ms. Gaganpreet Kaur, Mr. Akshay Girdhar and Ms. Manvjeet Kaur, " Article: Enhanced Iris Recognition System - an Integrated Approach to Person Identification", International Journal of Computer Applications, vol.8, no.1, pp.1-5, Published by Foundation of Computer Science, October 2010. 